\title{
Knowledge Support of Simulation Model Reuse
}

\author{
M. Valášek, P. Steinbauer, Z. Šika, Z. Zdráhal
}

This describes the knowledge support for engineering design based on virtual modelling and simulation. These are the results of the EC Clockwork project. A typical and important step in the development of a simulation model is the phase of reusing. Virtual modelling and simulation often use the components of previous models. The usual problem is that the only remaining part of the previous simulation models is the model itself. However, a large amount of knowledge and intermediate models have been used, developed and then lost. A special methodology and special tools have therefore been developed on support of storing, retrieving and reusing the knowledge from previous simulation models. The knowledge support includes informal knowledge, formal knowledge and intermediate engineering models. This paper describes the overall methodology and tools, using the example of developing a simulation model of Trijoint, a new machine tool.

Keywords: Simulation model, reuse, knowledge management, machine tool.

\section{Introduction}

This paper describes the application of the reuse methodology developed within the framework of the EU Clockwork project (Creating Learning Organisations with Contextualised Knowledge-Rich Work Artifacts). The presented reuse approach is based on knowledge support for engineering design based on virtual modelling and simulation. The use of Clockwork tools on an application example is shown for demonstration purposes. The target is to obtain an overview of the possibilities and functionality of the Clockwork methodology and the tools from the Clockwork ToolKit.

Two examples are given. These are based on examples from the Clockwork project trials. First, we describe the development of the simulation model of the Dyna-M machine tool, as collaborative development taking place from scratch between two geographically distributed partners. Then we describe the development of simulation model of the Trijoint machine tool as a reuse development where the simulation model of Dyna-M is reused. Both the examples are motivated by current highly innovative developments in the machine-tool industry. It is expected that the new generation of machine tools with increased productivity will be based on hybrid kinematics. The development of such machine tools requires overall comprehensive modelling and simulation of dynamic interactions between nonlinear kinematics, structural flexibility, drives and advanced control systems.

\section{Collaborative development of a simulation model of Dyna-M from scratch}

This simulation model is being developed from scratch. The development process involves collaboration between two partners. One partner (A) is competent in modelling mechanics \& control. The other partner (B) is competent in modelling of drives. They are geographically distributed and they cooperate through the internet. The development follows the methodology described in [1,2]. The methodology follows the subsequent formulation of the simulation problem in four worlds: real (initial problem formulation), conceptual (of the problem decomposition into components), physical (creation of a physical model in ideal objects) and simulation (a proper simulation model).

The problem in the real world is formulated above. The real world object is formed from a Real World System, Question and Solution (Fig. 1). The Real World System is the Dyna-M machine tool. The modelling goal is given by the question. The question concerns the dynamic interaction within the machine, in particular what level of dynamic accuracy can be achieved within interaction between nonlinear kinematics, structure flexibility, drive dynamics and control. The solution to this question will be answered by a simulation experiment on the simulation model.

This data is stored in the Clockwork Knowledge Management Tool (CKMT) (see Fig. 1). A new case is opened. The texts (abstracts), pictures and possible further extending documents in files (e.g. papers, reports) are filled in and downloaded into CKMT. This is the informal knowledge on the level of a Real World Object.

The formal knowledge is represented by semantic indexing. The Real World Object is named as "Machine tool Dyna-M", and it is classified in the product ontology (Fig. 2) as "horizontal-machine-tool". This semantic indices will later be used for search and retrieving of suitable cases. This is done by partner A, who acts as the leading partner in the cooperation.

The next step according to the Clockwork methodology is conceptualization. The conceptualization insists on decomposition of the Real World System into components. In many cases such a decomposition is described by a CAD drawing or of 3D volumetric geometric models. During this decomposition the first assumptions are raised, e.g. neglecting of parts of the machine. In our case, for example, the moving table, spindle motion are not considered. As a part of the decomposition, the Real World System is decomposed into the machine system (becoming the conceptual model) and the environment system (becoming the modeling environment) describing the interaction (excitation) of the system from outside. The question is formulated in greater detail and becomes the Modelling objective.

The conceptual model itself can be developed and described using any tool for graphical sketching. In our case it was exported from the CAD drawing into Corel Draw and 


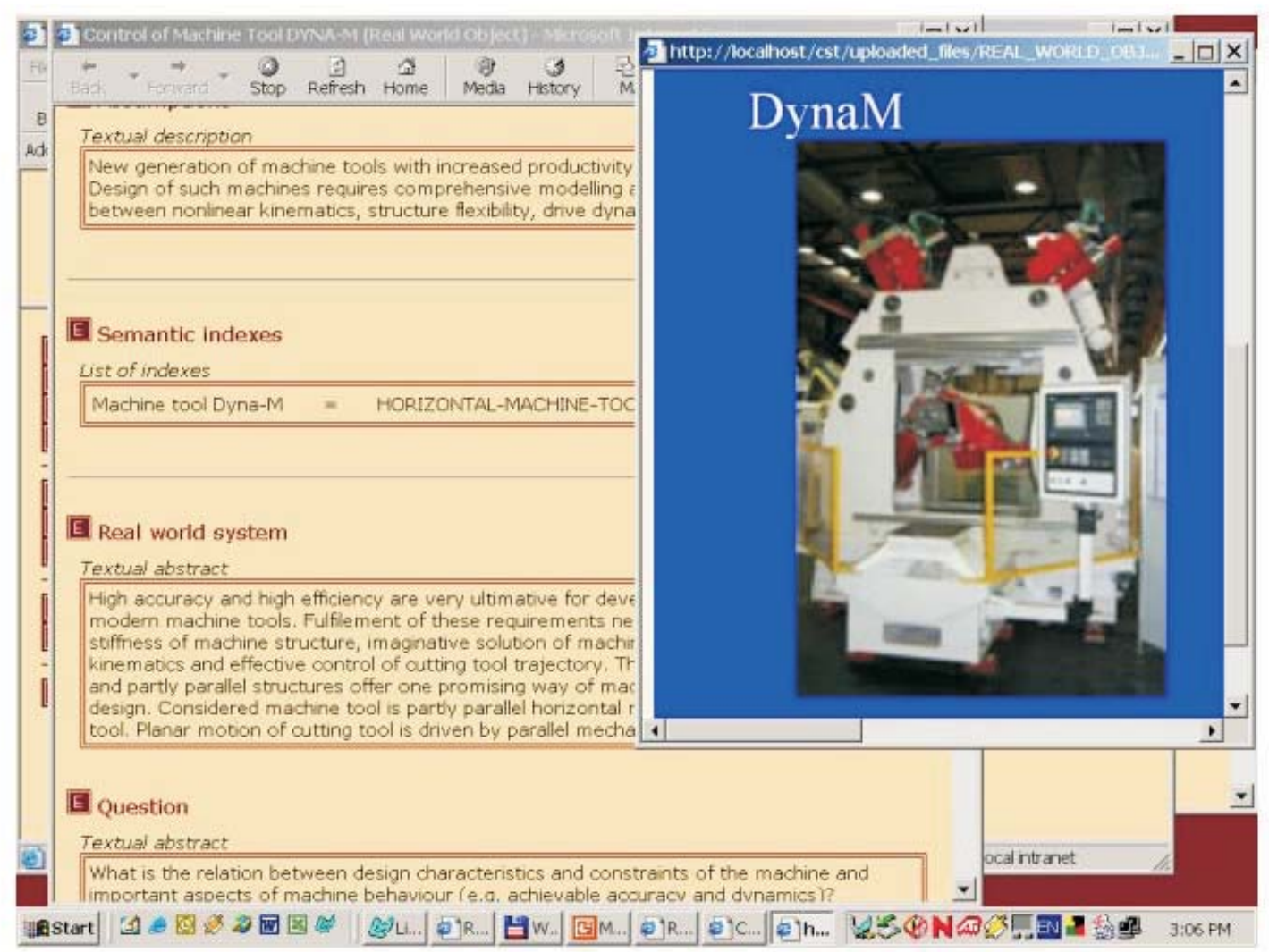

Fig. 1: Dyna-M as a Real World System in CKMT

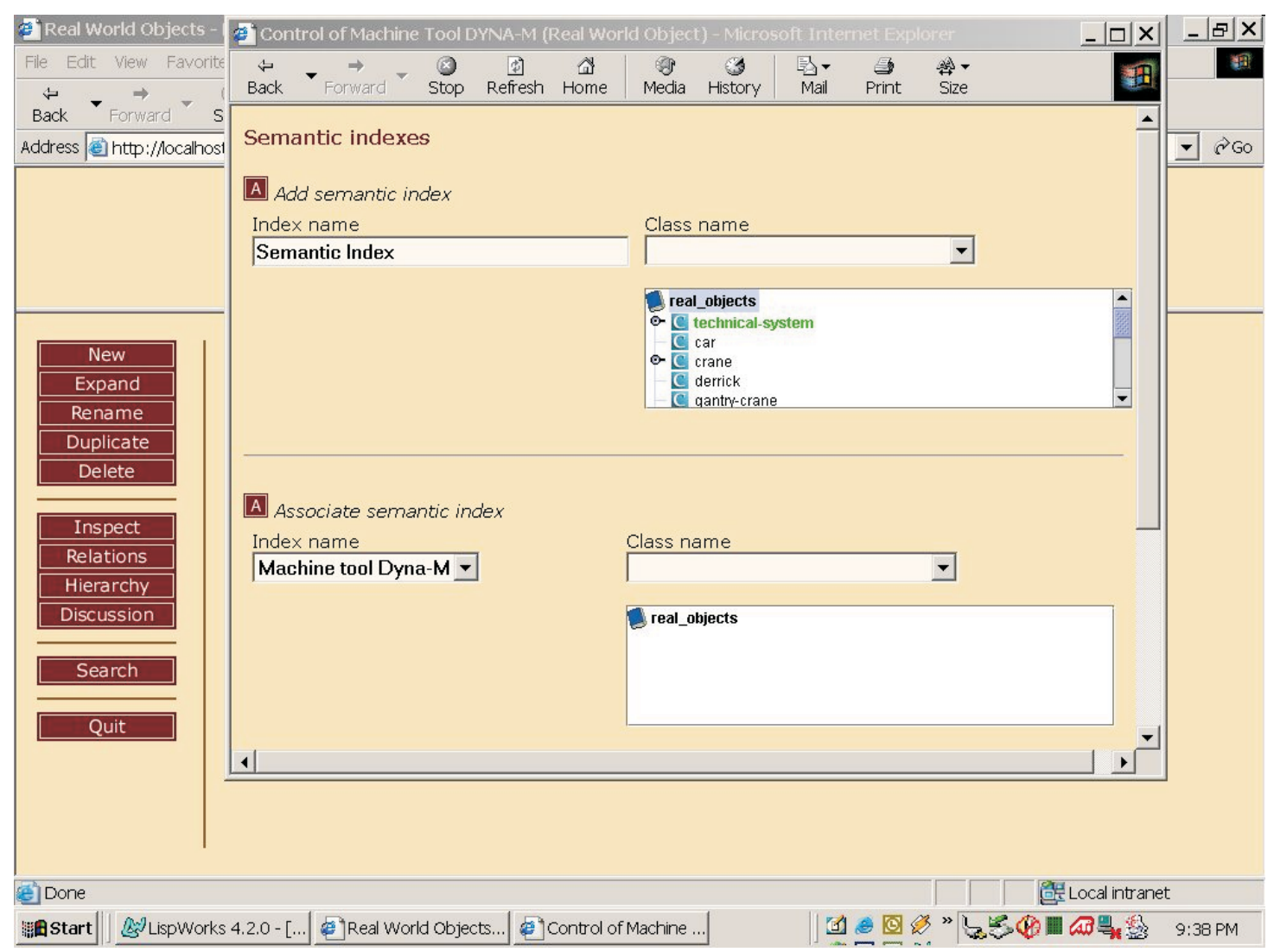

Fig. 2: Semantic indexing of the Dyna-M simulation problem 


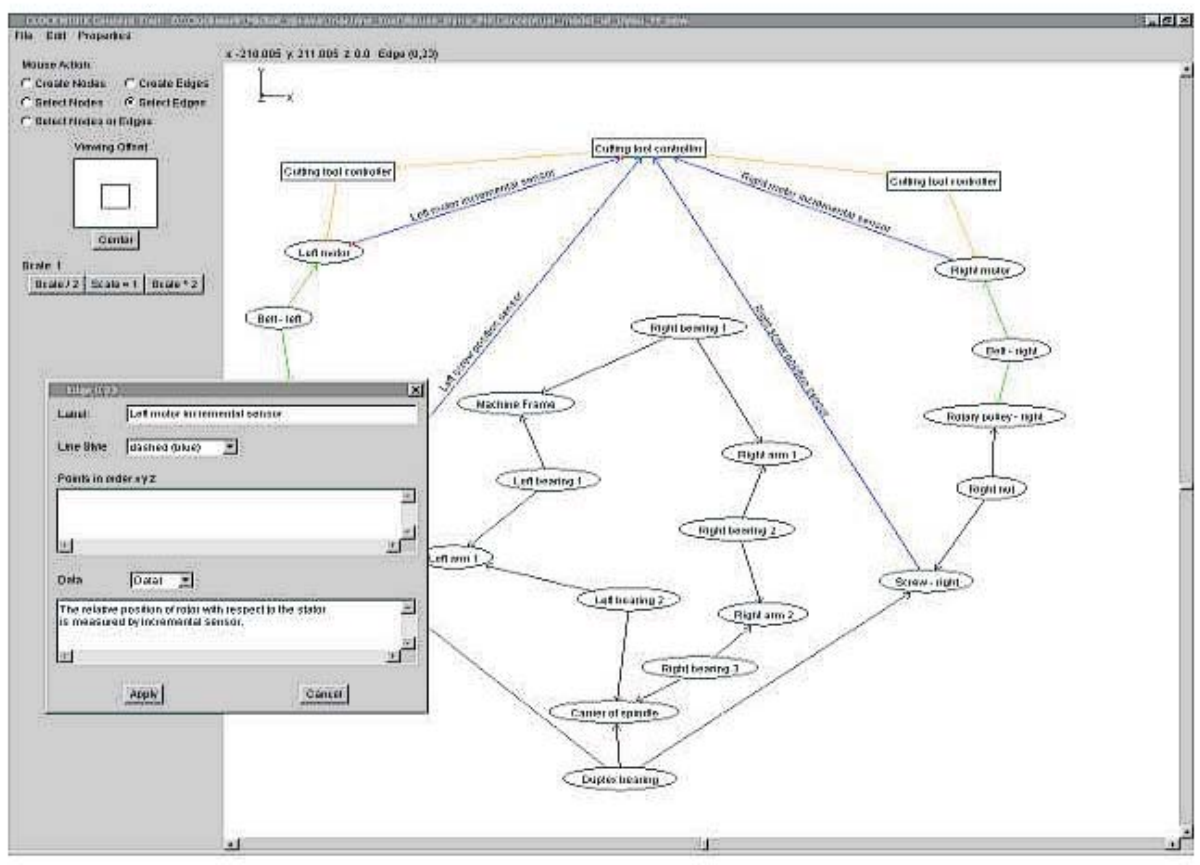

Fig. 3: Dyna-M conceptual model including annotations

finally into jpg format. The drawing was extended by the names of particular components for later semantic indexing. The development and description of the conceptual model can also be done within the Modelling tool from CLOCKWORK (Fig. 3). More support can be provided for saving appropriate knowledge. The modelling tool supports the annotations of particular components and automatic export of their names for semantic indexing.

The developed Conceptual World Object is stored into CKMT. A new case is opened. The texts (abstracts), pictures, files containing the conceptual model(s) and possible further extending documents in files (e.g. papers, reports) are filled in and downloaded into CKMT. This is the informal knowledge on the level of Conceptual World Object. The formal knowledge is again created by semantic indexing. The names of particular components are classified in the component ontology and stored in CKMT. These semantic indices will later be used for search and retrieving of suitable cases.

All this is done by partner A. Now the cooperation begins. The stored data in CKMT is transmitted to partner B. Using the accompanying e-mail discussion space or using e-mail directly, it is agreed that partner $\mathrm{B}$ will develop the modelling and simulation part of the electric drives. He studies and analyses the material in CKMT, discusses with partner A, and as a result he adds and/or modifies the data in CKMT on the level of Conceptual World Object. These iterations of Conceptual World Object are stored by partner B into CKMT either as a modification of an existing Conceptual World Object or, as a new version of it. As a result of these iterations, a Conceptual World Object is developed that is acceptable for both partners A and B. At this stage the semantic indexing can be completed. This refers the semantic indices themselves, but it refers especially to the description of the relation "is conceptualised as" between Real World Object and the Conceptual model.

The following step according to the Clockwork modeling and simulation methodology is proper modeling [1, 2]. The modeling insists on the subsequent replacement of components of the conceptual model by ideal objects, and thus obtaining the physical model. During this process the interfaces between partners A and B must first be specified. Then both partners develop physical models of their parts of the complete model. During this process many different modeling assumptions are raised, e.g. the links of Dyna-M are modeled as rigid bodies and the flexibility is taking into account in the models of the moving screws.

The modeling environment is transformed into the model input, and the modeling objective is transformed into the model output, i.e. into the excitation of the model and the measures for evaluating of the solution. The development and description of the physical model can also be done within the Modeling tool (Fig. 4), but any other graphical tool can be used. The modeling tool can provide more support for saving appropriate knowledge. The modeling tool supports the annotations of particular ideal objects and automatic export of their names for semantic indexing.

The developed Modeled World Object is stored into CKMT. A new case is opened. The texts (abstracts), pictures, files containing the physical model(s) and possible further extending documents in files (e.g. papers, reports) are filled in and downloaded into CKMT. This is the informal knowledge on the level of Modeled World Object. The formal knowledge is created again by semantic indexing. The names of particular components are classified in the ideal objects ontology and stored in CKMT. Then the description of the relation "is modeled as" between Conceptual and Physical models (Fig. 5) is also created. These semantic indices and relations will later be used for search and retrieving of suitable cases.

The next step according to the Clockwork modeling and simulation methodology is the simulation model implementation and later simulation experimentation. The simulation model implementation insists on the subsequent replacement of ideal objects in the physical model by the constructs of the simulation software, and thus obtaining the simulation 


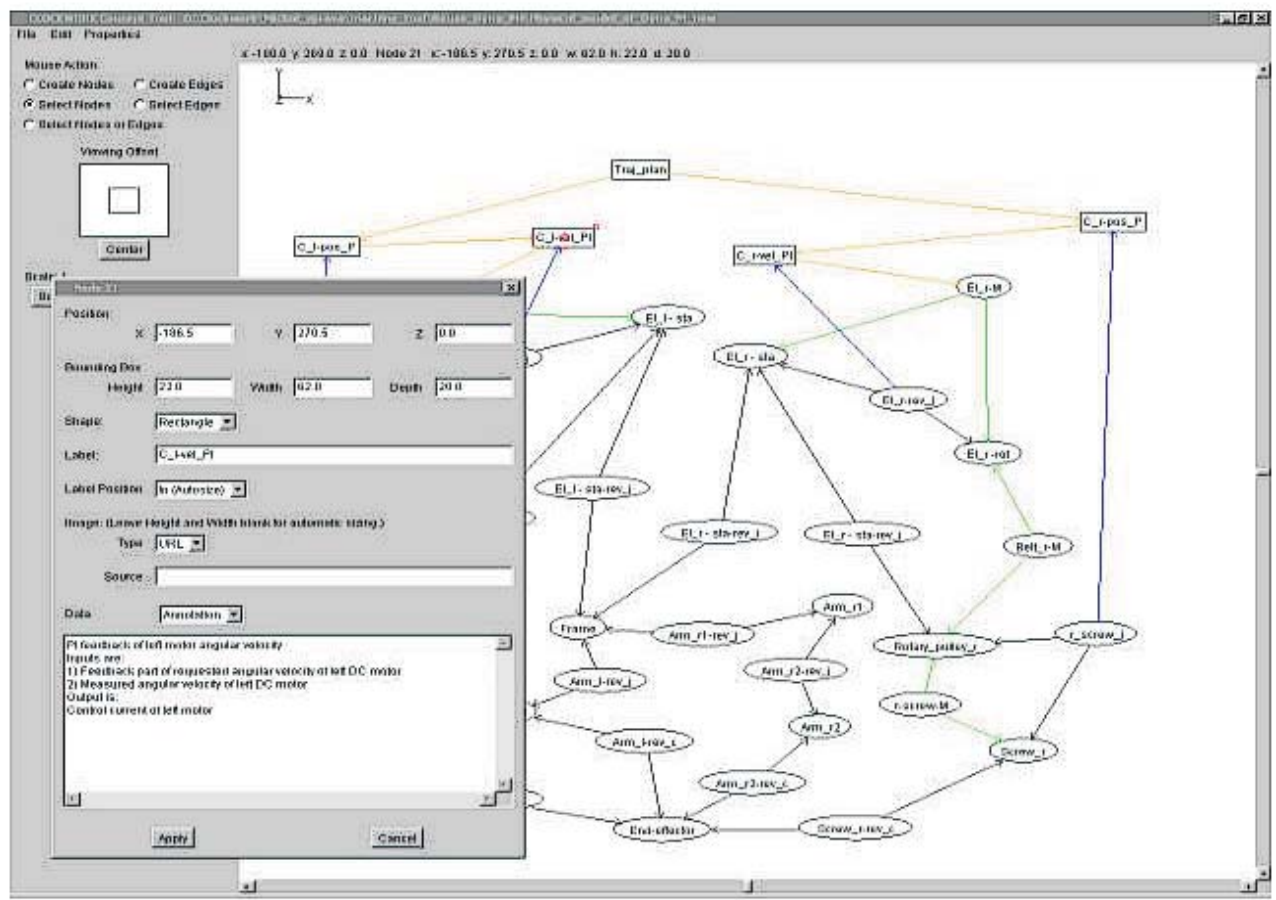

Fig. 4: Dyna-M physical model

model. Again the interfaces between partners A and B must be specified. Then both partners develop the simulation models of their parts of the complete model. For the solution of the simulation model a solution procedure must be selected. During this process further assumptions are raised, e.g. the chosen accuracy of the integration procedure. During the implementation, the testing model inputs and model outputs are used in order to test the correctness of the implementation during the testing simulation runs. In our case, for ex- ample, the unit steps are used in order to check the dynamic behaviour.

Implementation of the simulation model is realized in the simulation software (in our case in Matlab-Simulink), but the simulation annotation tool (Fig. 6) for saving appropriate knowledge is of great importance. The simulation annotation tool developed for Simulink supports the annotations of particular simulation constructs and automatic export of their names for semantic indexing.

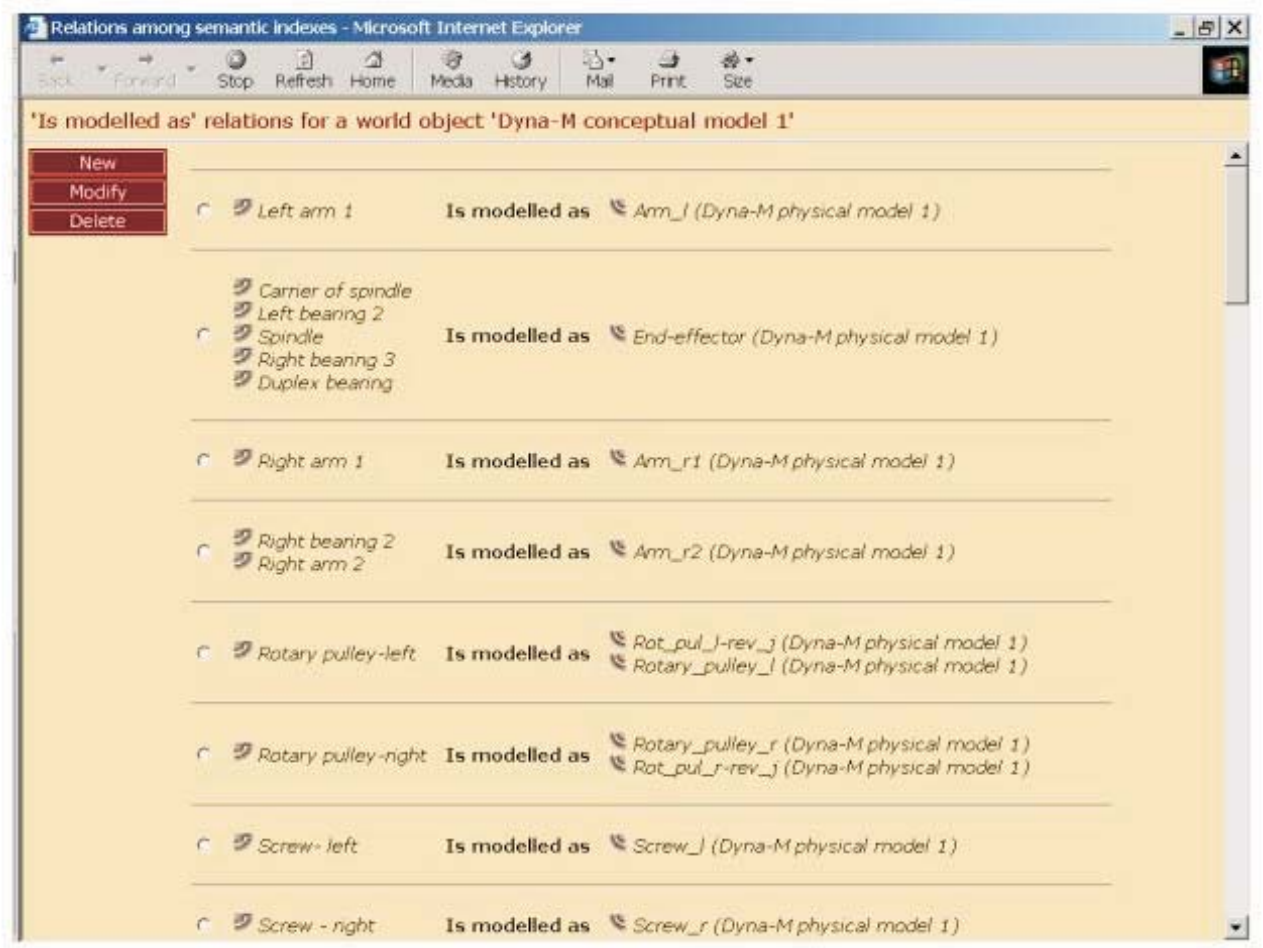

Fig. 5: Semantic indexing of relations between conceptual and physical models 


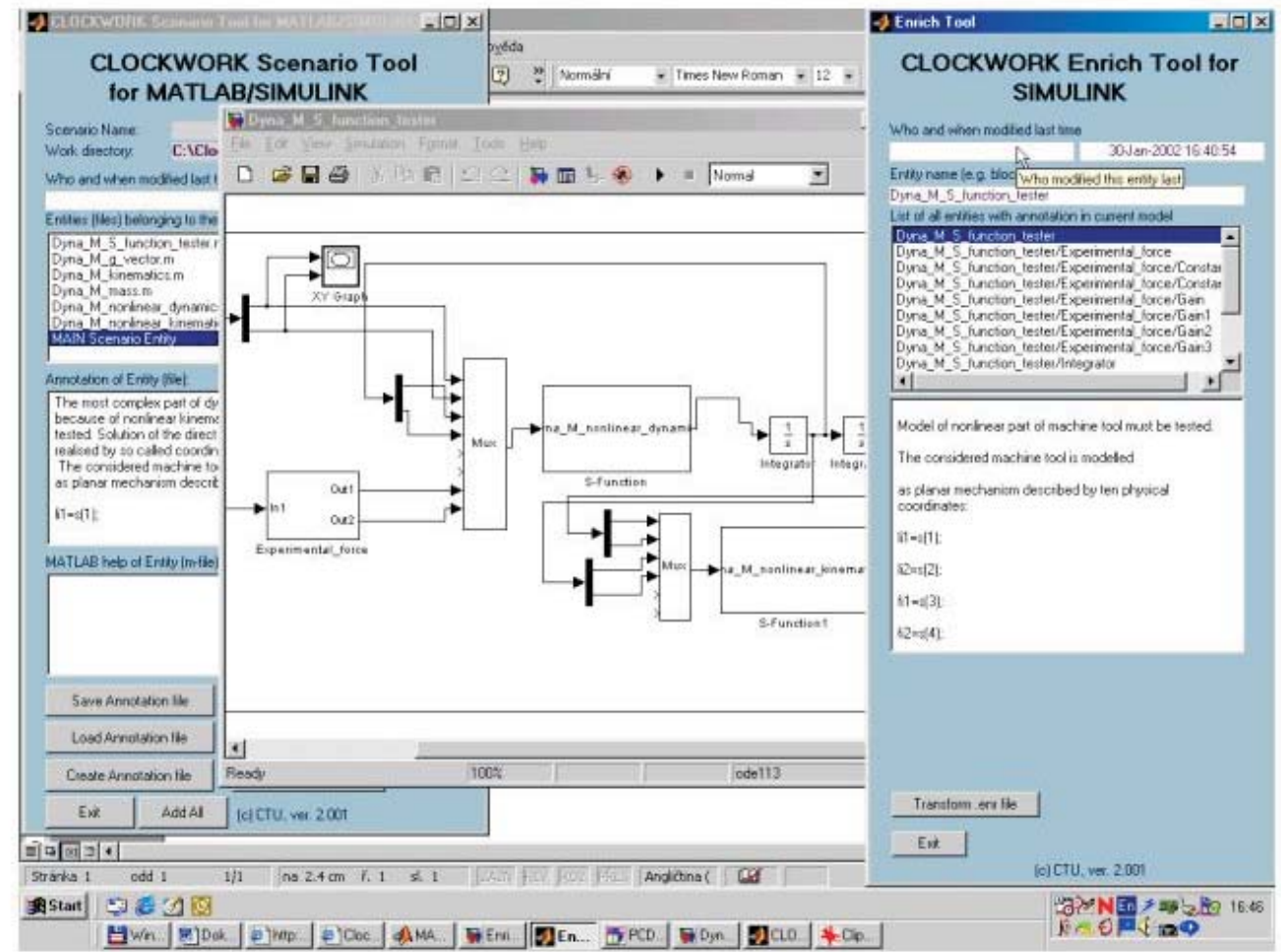

Fig. 6: Simulation model of Dyna-M with annotations

\section{Lessons learned from the Dyna-M case}

The developed Simulated World Object is stored into CKMT. The final step according to the Clockwork methodology is the simulation experiment. The tested simulation model is finally run with the model input developed on the level of a physical model. The model outputs are analyzed and interpreted in order to answer the question from a real world object. In the case of Dyna-M the real input was the motion along the desired circular trajectory, the achieved dynamic accuracy was investigated. The results of the simulation experiment are then stored in CKMT at the Simulated World Object and their interpretation is stored as the Solution at the Real World Object. This finally closes the life cycle of modeling and simulation according to the Clockwork methodology.

At this stage, after finishing the first example, the advantages of the Clockwork approach and the main differences between the Clockwork approach and the traditional development process of simulation models can be summarized. First, the additional supporting tools for modeling and simulation developed in the Clockwork project can be listed. Leaving aside the knowledge part (Clockwork knowledge management tool, Appolo ontology tool, ontology interface tool), there are tools that are applicable and useful when applied alone. These are the Simulation Annotation Tool and the Modeling Tool. They support the description of different models created within the development process of the simulation model.

Regarding the differences and advantages of the Clockwork approach over the traditional development process of a simulation model, the most important consideration is the amount of remaining work documents after the end of the development process. In the domain of engineering all models are fully developed including intermediate models that have been used during the development. In the domain of knowledge both informal and formal knowledge associated with the simulation development have been captured. The advantage of the Clockwork approach for the development of the cooperative simulation model is the fact that traditionally there has usually remained only the resulting simulation model without previous and associated tacit models and knowledge.

\section{Development of reuse of the simulation model of TriJoint machine-tool}

The second modeling and simulation case investigates the dynamic properties of the new machine tool Trijoint $900 \mathrm{H}$ (Fig. 7). This machine tool has been developed within cooperation between Kovosvit MAS and the Czech Technical University in Prague. It is a new machine tool concept patented worldwide by Kovosvit MAS and the Czech Technical University. It is also a machine tool with hybrid kinematics. Its remarkable property is that it has simultaneously increased both the dynamics and the stiffness by a factor of 2-3 over traditional machine tools.

The development is being done by partner $\mathrm{A}$, and this development will be supported by the reuse methodology and the capability of the Clockwork modeling and simulation methodology and the Clockwork Toolkit. First, the problem in the Real World is formulated. The Real World Object is developed with the real world system, question and expected solution. 


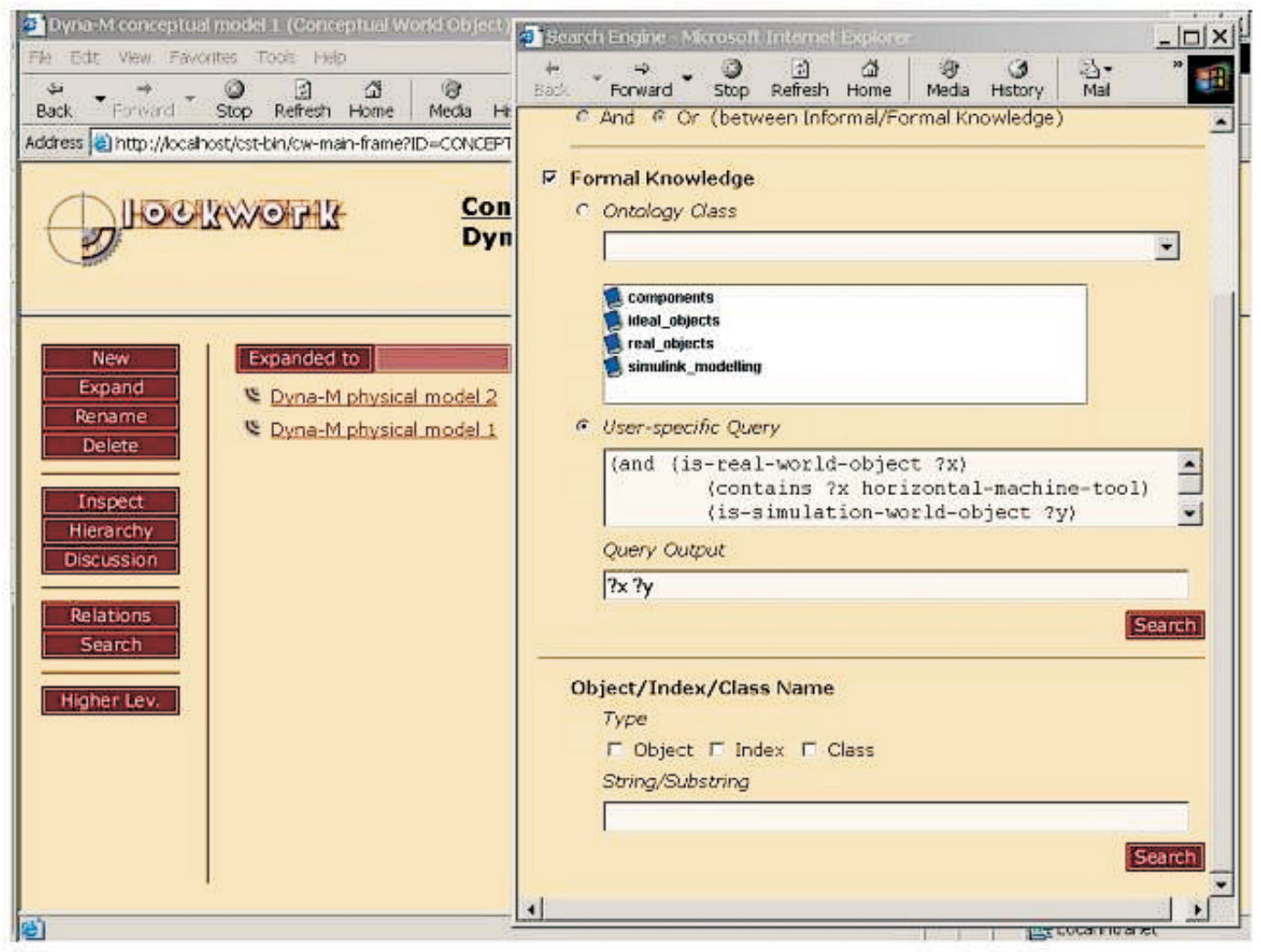

Fig. 7: Search for a similar case to TriJoint $900 \mathrm{H}$ using semantic indices

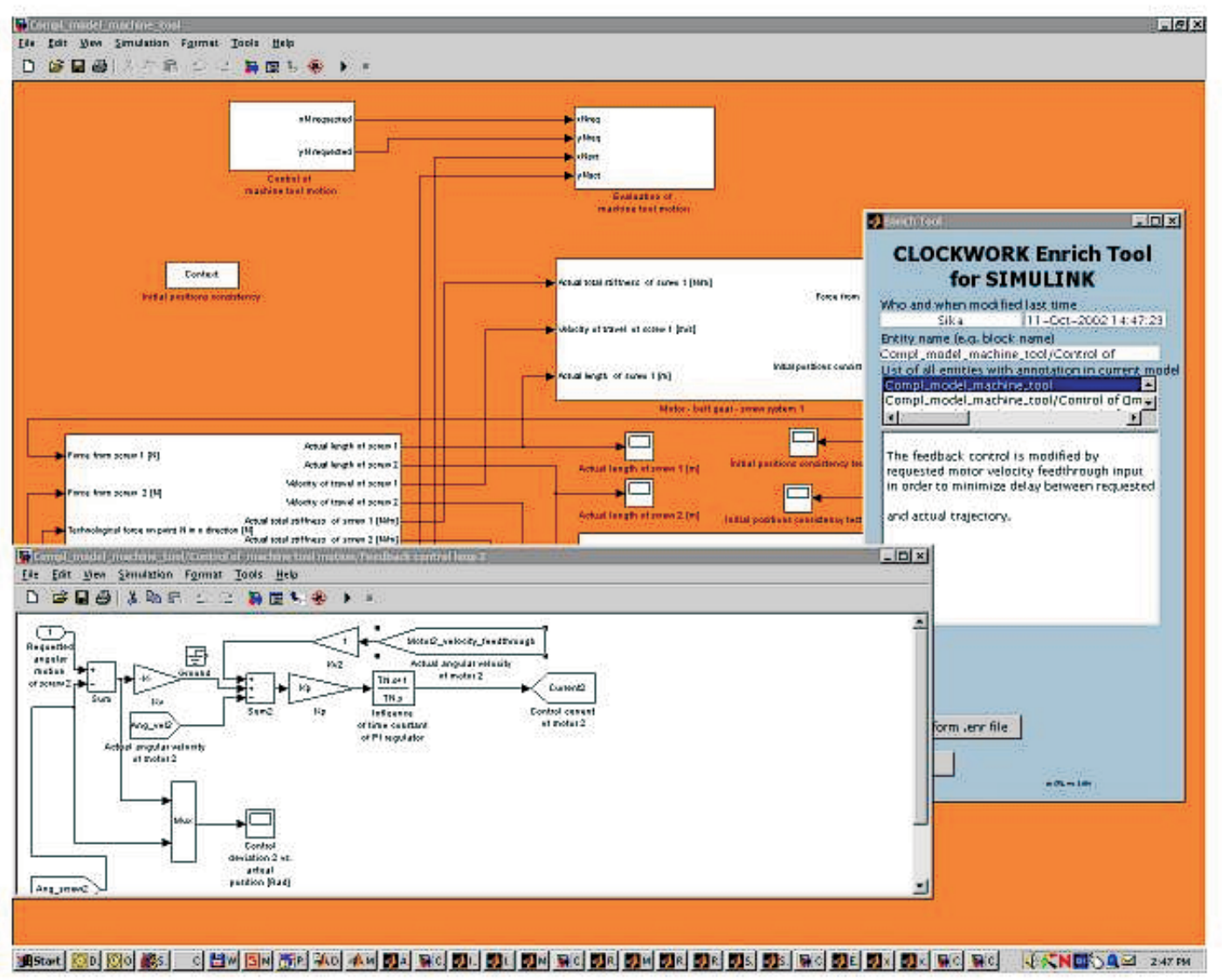

Fig. 8: Modified simulation model of TriJoint based on the reused simulation model of Dyna-M 
The resulting data is stored into CKMT similarly as for Dyna-M. A new case is opened. The texts (abstracts), pictures and possible further extending documents in files (e.g. papers, reports) are filled in and downloaded into CKMT. This is the informal knowledge on the level of Real World Object. The formal knowledge is created by semantic indexing. The Real World Object is named as "Machine tool PH" (PH, i. e., Parallel Horizontal machine tool was the working name before the final name TriJoint $900 \mathrm{H}$ ), and it is classified in the product ontology as "horizontal-machine-tool". This is stored in CKMT.

Based on this semantic indexing, the possibility arises of reusing some previous case from the CKMT database. This can be done by a search (Fig. 7) based on semantic indices. The search can be based on formal knowledge or even on informal knowledge. The search is based on looking for a similarity. The similarity in formal knowledge is based on a similarity in ontology. The similarity in informal knowledge is based on similarity with words of natural language (search through key words). The retrieved similar case from CKMT is Dyna-M.

The reuse development process within the Clockwork methodology can start. First the retrieved case of Dyna-M is copied into the PH case on the level of conceptual, modeling and simulation world objects.

Subsequently these levels are modified. First the conceptual model is modified and replaced in CKMT. The modeling tool for supporting modeling on levels above the simulation model is advantageously applied. The same is done on the physical modeling level with a physical model and MWO stored in CKMT. The final level of the simulation model is solved by replacing of blocks of dynamics and kinematics of Dyna-M (Fig. 6) with the corresponding blocks for $\mathrm{PH}$ (TriJoint) (Fig. 8). The resulting Simulation World Object is stored in CKMT.
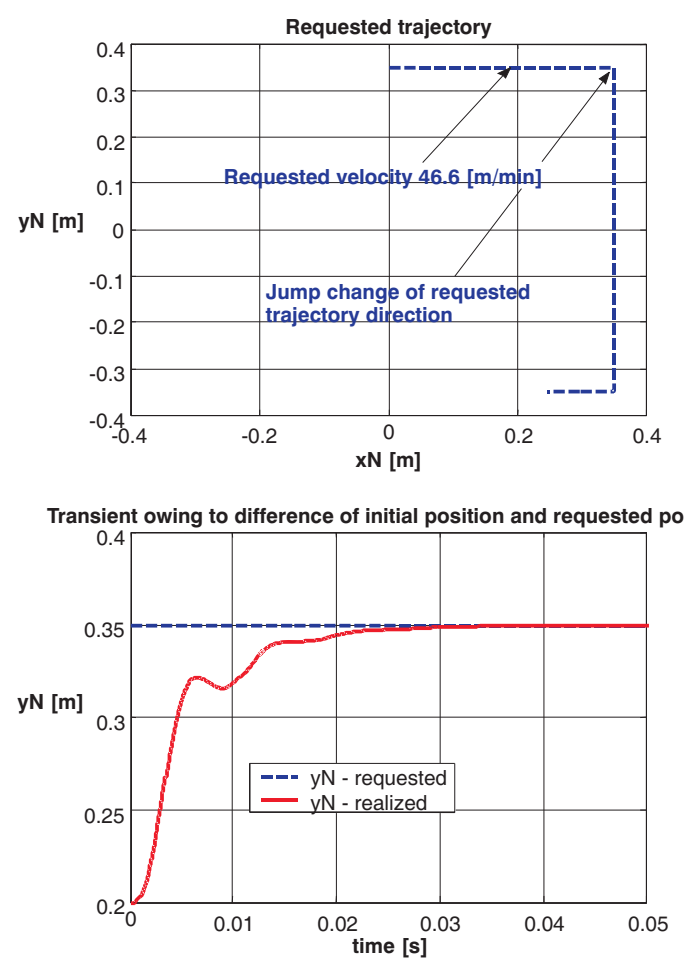

The more material that can be reused the more important is the testing of the implemented simulation model (Fig. 9). After its successful realization, simulation experiments can be provided. The results of the simulation experiment are then stored in CKMT at the Simulated World Object, and their interpretation is stored as the Solution at the Real World Object. This finally again closes the life cycle of modeling and simulation according to the Clockwork modeling and simulation methodology.

\section{Lessons learned from the TriJoint case}

Regarding the differences and advantages of the Clockwork approach over the traditional development process of a simulation model, it may be stated that the reuse of traditional simulation models is much more difficult compared with Clockwork approach. In traditional approach there do not exist abstract models, informal knowledge, especially annotations of all models. This support for proper understanding of the simulation model is usually impossible, thus preventing reuse alltogether. The main supporting aspects for modeling and simulation developed with the Clockwork project with special importance for reuse can be listed, as follows:

- Use of the facility for knowledge sharing in collaboration and reuse has been demonstrated.

- The domain of engineering supported and organized on the basis of support for the knowledge domain.

- A semantics based search through:

o formal knowledge,

o informal knowledge,

o reasoning of both.
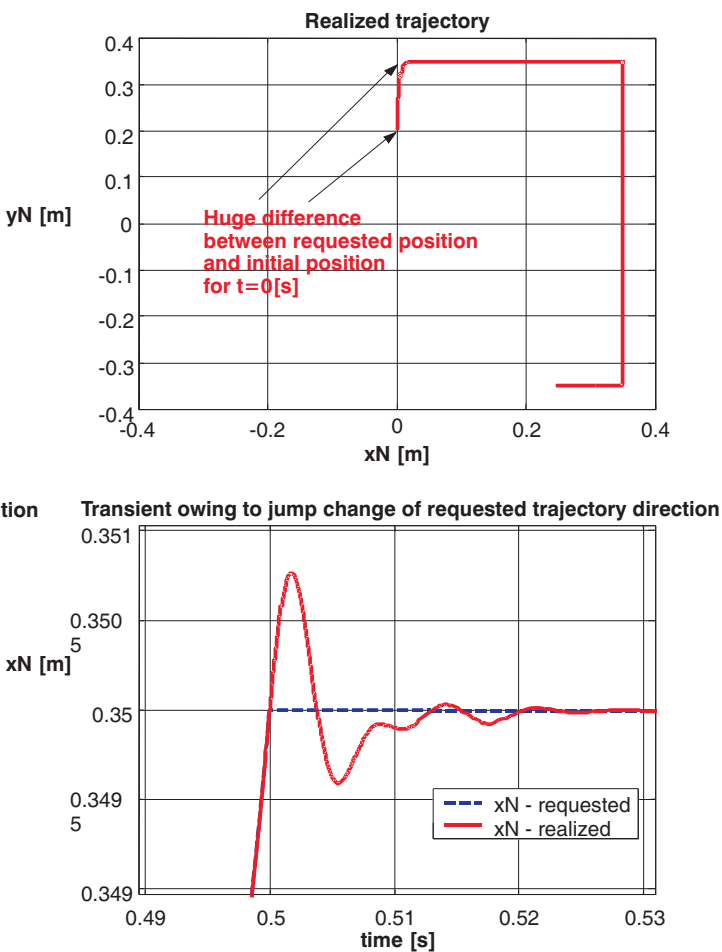

Fig. 9: Simulation experiments with the simulation model of TriJoint $900 \mathrm{H}$ 


\section{Conclusions}

The Clockwork approach including the Clockwork methodology and tools can be evaluated on the basis of experience with development of the simulation model of Dyna-M and TriJoint. It can be concluded that the Clockwork approach supports both collaborative development and the reuse of simulation models. The Clockwork tools support all stages of the development process in the domain of both engineering and knowledge. Finally, it can be concluded that the Clockwork approach improves the whole engineering process of modeling and simulation.

\section{Acknowledgment}

The authors appreciate the support provided by the EU IST 12566 project CLOCKWORK.

\section{References}

[1] Steinbauer P., Valášek M., Zdráhal Z., Mulholland P., Šika Z.: "Knowledge Support of Virtual Modelling and Simulation", in: Proc. of NATO ASI Virtual Nonlinear Multibody Systems, Prague 2002, p. II/241-246.

[2] Valasek M., Steinbauer P., Kolar J., Dvorak J.: “Concurrent design of railway vehicles by simulation model reuse", this AED 03 proceedings.

Prof. Ing. Michael Valášek, DrSc.

phone: +420224357361

fax: +420224916709

e-mail: michael.valasek@fs.cvut.cz

Ing. Pavel Steinbauer, Ph.D.

e-mail: pavel.steinbauer@fs.cvut.cz

Ing. Zbyněk Šika, Ph.D.

e-mail: zbynek.sika@fs.cvut.cz

Department of Mechanics

Czech Technical University in Prague

Faculty of Mechanical Engineering

Karlovo nám. 13

12135 Praha 2, Czech Republic

Prof. Zdeněk Zdráhal

Knowledge Media Institute

The Open University

Walton Hall

Milton Keynes, UK 